\title{
Construções simbólicas do tratamento de pessoas que vivem com o HIV/AIDS entre enfermeiros
}

\author{
Nurses' symbolic construction of treatment for people living with HIV/AIDS \\ Construcciones simbólicas del tratamiento de personas que viven con VIH/SIDA entre enfermeros
}

\author{
Denize Cristina de Oliveira; Thelma SpindolaII; Antonio Marcos Tosoli Gomes ${ }^{I I I}$; Sergio Correa Marques ${ }^{I V}$; \\ Glaucia Alexandre Formozo ${ }^{V}$; Letícia de Araújo Campos ${ }^{\mathrm{VI}}$
}

\begin{abstract}
RESUMO: A Síndrome da Imunodeficiência Adquirida (AIDS) no Brasil encontra-se disseminada em diferentes grupos populacionais, determinando construções sociocognitivas frente ao tratamento. O estudo objetivou analisar as representações sociais de enfermeiros sobre tratamento de pessoas que vivem com o Vírus da Imunodeficiência Humana (HIV)/AIDS. Estudo qualitativo, realizado em 2010, em hospital público universitário no Rio de Janeiro, com 20 enfermeiros que responderam a entrevistas semiestruturadas, submetidas à análise de conteúdo temático-categorial. Observou-se uma rede de representações entre os enfermeiros na qual o tratamento da pessoa que vive com HIV/AIDS é reconhecido nas suas dimensões física, psicossocial e relacional, a partir de categorias que não se reduzem à terapia medicamentosa, incorporando os cuidados clínicos e físicos, os sentimentos, as facetas social, relacional e psicológica do tratamento. As representações sociais do tratamento assumem um papel importante no cenário do cuidado em saúde e compreendem as ações reconhecidas pelos enfermeiros para o controle da AIDS, participando da determinação das práticas de cuidado desenvolvidas.
\end{abstract}

Palavras-Chave: Tratamento; HIV/AIDS; representação social; enfermeiro.

ABSTRACT: AIDS is present in different population groups in Brazil, determining socio-cognitive constructions as regards treatment. This qualitative study analyzed nurses' social representations of treatment for people living with HIV/AIDS by semi-structured interview of 20 nurses at a public university hospital in 2010 in Rio de Janeiro, and analysis of the transcripts for thematic content. There was a network of representations among the nurses in which treatment for people living with HIV/AIDS was recognized in its physical, psychosocial and relational dimensions, on the basis of categories that do not reduce to drug therapy, but include clinical and physical care, feelings, and social, relational and psychological aspects of treatment. Social representations of treatment play an important role in the care setting: they encompass the actions recognized by nurses to control AIDS, and thus participate in determining the care practices applied.

Keywords: Treatment; HIV/AIDS; social representation; nurse.

RESUMEN: La Síndrome de Inmunodeficiencia Adquirida (SIDA) en Brasil está difundida en medio a diferentes grupos de la población, determinando construcciones sociocognitivas ante el tratamiento. El estudio tuvo el objetivo de analizar las representaciones sociales de enfermeros sobre el tratamiento de personas que viven con el Virus de la Inmunodeficiencia Adquirida (VIH)/SIDA. Estudio cualitativo, desarrollado en 2010, en un hospital público universitario de Rio de Janeiro-Brasil, junto a 20 enfermeros que contestaron entrevistas semiestructuradas, analizadas a través de la técnica de análisis de contenido temático y categorial. Los resultados muestran una red de representaciones entre los enfermeros en la que el tratamiento de la persona que vive con $\mathrm{VIH} / \mathrm{SIDA}$ se reconoce en sus dimensiones física, psicosocial y relacional, a partir de categorías que no se reducen a la terapia medicamentosa, sino que incorporan los cuidados clínicos y físicos, los sentimientos y las facetas social, relacional y psicológica del tratamiento. Las representaciones sociales del tratamiento juegan un papel importante en el cuidado en salud e incorporan las acciones reconocidas por los enfermeros para el control del SIDA, además de participar en la determinación de las prácticas de atención desarrolladas. Palabras Clave: Tratamiento; VIH/SIDA; representación social; enfermero.

\section{INTRODUÇÃO}

O Vírus da Imunodeficiência Humana (HIV) e a Síndrome da Imunodeficiência Adquirida (AIDS) estão disseminados em todos os grupos populacionais no Brasil, apresentando grupos sociais de maior vul- nerabilidade social. $\mathrm{O}$ perfil epidemiológico sinaliza a ocorrência da heterossexualização, feminização, juvenização e pauperização da epidemia, havendo aumento de casos entre grupos com baixa escolaridade,

${ }^{\mathrm{I}}$ Doutora em Enfermagem. Professora Titular do Departamento de Fundamentos de Enfermagem da Faculdade de Enfermagem da Universidade do Estado do Rio de Janeiro. Brasil. E-mail: dcouerj@gmail.com.

IIDoutora em Enfermagem. Professora Associada do Departamento de Fundamentos de Enfermagem da Faculdade de Enfermagem da Universidade do Estado do Rio de Janeiro. Brasil. E-mail: tspindola.uerj@gmail.com.

IIIDoutor em Enfermagem. Professor Titular do Departamento de Enfermagem Médico-Cirúrgica da Faculdade de Enfermagem da Universidade do Estado do Rio de Janeiro. Brasil. E-mail: mtosoli@gmail.com.

${ }^{\text {IV }}$ Doutor em Enfermagem. Professor Adjunto do Departamento de Fundamentos de Enfermagem da Faculdade de Enfermagem da Universidade do Estado do Rio de Janeiro. Brasil. E-mail: scmarques@uol.com.br.

vDoutora em Psicologia Social. Professor Adjunto da Escola de Enfermagem - Campus Macaé da Universidade Federal do Rio de Janeiro. Macaé, Rio de Janeiro, Brasil. E-mail: glaucinhaenf@yahoo.com.br.

${ }^{\mathrm{V}}$ Mestre em Enfermagem pela Faculdade de Enfermagem da Universidade do Estado do Rio de Janeiro. Primeiro-Tenente da Marinha do Brasil. Rio de Janeiro, Brasil. E-mail: leticiauffenf@gmail.com. 
mulheres, jovens e negros ${ }^{1}$. Considera-se, no entanto, que todos os grupos são vulneráveis à infecção pelo HIV e a suscetibilidade será maior de acordo com o contexto social e os níveis de exclusão socioculturais e econômicos ${ }^{2}$.

Desde o seu início até 2014, a epidemia da AIDS registrou 734 mil casos no Brasil, o que representa uma taxa de prevalência de $0,4 \%$ da população geral. A maior concentração dos casos está entre os indivíduos entre 25 e 39 anos de ambos os sexos, sendo 54\% do total de casos entre os homens. A taxa de detecção no país tem apresentado estabilização nos últimos dez anos, com uma média de 20,5 casos para 100 mil habitantes, com maior tendência de queda na Região Sudeste $(28,1 \%)$, passando de 26 para 18,7 casos para 100 mil habitantes. O coeficiente de mortalidade passou de 6,1 em 2004 para 5,7 óbitos para $100 \mathrm{mil}$ habitantes em 2013, representando uma queda de 6,6\% nas Regiões Sudeste e Sul. Os Estados do Rio de Janeiro e Amazonas representam o segundo e o terceiro maiores coeficientes de mortalidade do país. Do total de óbitos por AIDS, 71,3\% ocorreram entre homens e 28,6\% entre as mulheres. Observa-se um aumento da sobrevida à medida que o diagnóstico da infecção é realizado precocemente ${ }^{1,3}$.

A introdução da terapia antirretroviral (TARV) reduziu a mortalidade associada à infecção pelo HIV/ AIDS, diminuindo o número de internações hospitalares e a ocorrência de infecções oportunistas, fazendo com que a AIDS passasse a ser considerada uma doença crônica ${ }^{1}$.

O impacto da TARV no aumento da expectativa e da qualidade de vida das pessoas que vivem com HIV/AIDS (PVHA) teve como um dos seus resultados o estabelecimento de um foco clínico e medicamentoso predominante do tratamento, com ações dirigidas ao diagnóstico, controle laboratorial das cargas virais, esquemas terapêuticos baseados exclusivamente em drogas e controle da transmissão. Mesmo reconhecendo a importância dessas ações para a redução da epidemia, o impacto social e psicológico da doença e do vírus, bem como as ações para o seu enfrentamento, não foram priorizados nas políticas públicas de combate à doença.

Considera-se que as tecnologias que compõem o tratamento das pessoas que vivem com o HIV/AIDS se apresentam como modalidade de cuidado de saúde, configurando-se como objeto de representações, uma vez que estão presentes na conversação cotidiana, desencadeando diferentes posicionamentos dos profissionais e clientes frente a elas.

Diante da problemática das diferentes concepções de tratamento destinado às PVHA, o objetivo deste artigo foi descrever e analisar as representações sociais das ações que compõem o tratamento das pessoas que vivem com o HIV/AIDS entre enfermeiros.

\section{REFERENCIAL TeÓRICO}

O cuidado é fundamental em ações que visam à promoção, manutenção e recuperação da saúde, sendo a principal tecnologia que a enfermagem utiliza para assistir o cliente de forma integral. Para atender às necessidades da PVHA, devem ser utilizadas tecnologias clínicas, psicológicas e sociais, orientadas pelo saber especializado da equipe multiprofissional, e apoiadas pela organização dos serviços e alocação de recursos ${ }^{4}$. Na prestação de assistência à PVHA, o enfermeiro enfrenta desafios de ordem política, social, cultural, econômica, bem como construções simbólicas que envolvem o profissional, seu grupo de pertença, o cliente e a família.

Cuidar de uma PVHA exige uma visão integral do ser humano, que apresenta demandas específicas, e se encontra fragilizado, merecendo respeito e atenção. Esse cuidado deve ser resgatado nas relações cotidianas e no pensar e fazer enfermagem, com emprego de tecnologias e da sensibilidade nas relações pessoais e profissionais ${ }^{5}$.

Cuidar de PVHA representa um desafio para os profissionais de saúde, em função do caráter discriminatório e estigmatizante da doença, ainda hoje presentes, e das dificuldades associadas à estrutura do sistema de saúde e das relações de trabalho no setor ${ }^{6}$.

Observa-se a existência de três dimensões do cuidado disponibilizado pelos profissionais de saúde ao assistir PVHA, nomeadas como cognitiva, afetiva-relacional e técnico-instrumental, que se manifestam desde a revelação do diagnóstico e perduram ao longo do tratamento, até a fase de terminalidade ${ }^{7}$. Considera-se, ainda, que os conceitos de necessidades humanas e de saúde se encontram subjacentes às representações de cuidado de saúde, da mesma forma que permanece subjacente às práticas de saúde em geral ${ }^{8}$. O cuidado de saúde é visto como uma tecnologia de trabalho que se organiza de acordo com cada período histórico, enraizando-se em determinadas definições de necessidades e objetivando-se em processos de trabalho específicos, além de fazer parte de diferentes redes de representações que circundam a doença, a pessoa doente e o seu tratamento e enfrentamento 9 .

Considerando a complexidade e o forte caráter simbólico da doença e do vírus, bem como do próprio cuidado de saúde, esta pesquisa foi orientada pela Teoria de Representações Sociais, concebida na Psicologia Social. Considera-se que as representações sociais não são criadas por um único indivíduo, mas por grupos sociais mediante processos de comunicação e de cooperação ${ }^{10}$. Quando criadas, as representações "adquirem uma vida própria, circulam, se encontram, se atraem e se repelem e dão oportunidade ao nascimento de novas representações, enquanto velhas representações morrem"10:213.

Os sentidos e conteúdos representados diferenciam-se dentro de uma mesma sociedade e da mesma cultura e esta diferenciação deve ser observada de 
acordo com o modo de pensar e de compreender desta sociedade. Os marcadores dessas diferenças são "as especificidades do universo consensual e do universo reificado e os contextos da comunicação em que essas representações são elaboradas"10:213.

Enquanto forma de conhecimento do senso comum, e também modalidade particular do conhecimento técnico-profissional, a representação social é entendida a partir do contexto em que está inserida e da sua funcionalidade no cotidiano das relações sociais, "articulando elementos afetivos, mentais, sociais, integrando a cognição, a linguagem e a comunicação às relações sociais que afetam as representações sociais e à realidade material, social e ideativa sobre a qual elas intervêm"11:41.

\section{Metodologia}

Estudo de natureza descritiva, qualitativa, na qual a coleta de dados ocorreu em 2010, em um hospital público universitário do Município do Rio de Janeiro, com 20 enfermeiros que cuidavam de PVHA, atuando nos setores de doenças infectoparasitárias, clínica médica, pneumologia e centro de tratamento intensivo geral.

A escolha dos participantes do estudo foi por conveniência e associada ao desempenho de atividade profissional afeita ao objeto de investigação. Assim, os enfermeiros que cuidavam de PVHA nos setores já citados foram convidados a participar da pesquisa. Aqueles que concordaram em participar responderam a uma entrevista semiestruturada e a um questionário de caracterização socioeconômico-profissional, abordando as seguintes variáveis: idade, sexo, renda mensal, estado marital, número de filhos, função exercida e tempo de atuação, formação profissional, tempo de formação profissional e tempo de atuação profissional com PVHA.

Os depoimentos dos enfermeiros foram analisados pela aplicação da técnica de análise de conteúdo temático-categorial, que apresenta as seguintes etapas: pré-análise, exploração do material, tratamento dos resultados, inferência e respectiva interpretação ${ }^{12,13}$.

A pesquisa foi aprovada por um Comitê de Ética com o parecer $\mathrm{n}^{\mathrm{O}} 1575$ e os profissionais assinaram o Termo de Consentimento Livre e Esclarecido. Para preservar o anonimato dos participantes, as entrevistas receberam a identificação E1, E2, E3... à medida que se sucediam.

\section{Resultados e Discussão}

A caracterização dos entrevistados revela que a maioria é do sexo feminino (15/75\%); com idades entre 35-44 anos e igual ou superior a 45 anos (9/45\%); vive com companheiro (13/65\%); tem filhos $(13 / 65 \%)$; renda mensal entre $\mathrm{R} \$ 3.000,00$ a 6.000,00 (14/70\%); tem pós-graduação lato sensu (15/75\%); trabalha há mais de 10 anos na enfermagem (19/95\%); atua entre
11-20 anos no hospital investigado (12/60\%); trabalha há mais de 20 anos na função de enfermeiro (10/50\%); e há 15 anos ou mais com PVHA (11/55\%).

A análise de conteúdo resultou em 541 unidades de registro (UR), distribuídas em 160 unidades de significação. A agregação dessas unidades resultou em seis categorias apresentadas na Tabela 1.

TABELA 1: Categorias, unidades de registro e unidades de significação do tratamento de PVHA entre os enfermeiros estudados. Rio de Janeiro, 2007.

\begin{tabular}{lccc}
\hline Categorias de tratamento & US $^{(*)}$ & UR $^{(* *)}$ & \% UR \\
\hline Cuidados clínicos e físicos & 31 & 166 & 30,7 \\
Tratamento medicamentoso & 49 & 111 & 20,5 \\
Tratamento psicoemocional- & & & \\
espiritual & 23 & 95 & 17,6 \\
Cuidado relacional & 15 & 60 & 11,1 \\
Terapêutica social & 25 & 59 & 10,9 \\
Ações terapêuticas educativas & 17 & 50 & 9,2 \\
Total & 160 & 541 & 100,0 \\
\hline
\end{tabular}

${ }^{(*)} U S$ - unidade de significação; ${ }^{(* *)} U R$ - unidade de registro.

As seis categorias definidoras do tratamento das PVHA para os enfermeiros estudados foram: Cuidados clínicos e físicos; Tratamento medicamentoso; Tratamento psicoemocional-espiritual; Cuidado relacional; Terapêutica social e Ações terapêuticas educativas, descritas a seguir.

\section{Cuidados clínicos e físicos}

O tratamento clínico da PVHA tem como um dos seus componentes principais o monitoramento da carga viral, que consiste na avaliação do sistema imunológico por meio do comparecimento periódico ao serviço de saúde para a realização de consulta médica destinada à avaliação dos resultados dos exames laboratoriais. As UR abaixo exemplificam essa constatação:

É importante explicar a necessidade de fazer os exames periodicamente. (E12)

O sistema imunológico do doente está comprometido, já pela própria doença, um foco de infecção pode ser absurdamente fatal. (E17)

A falha no acompanhamento laboratorial e aumento da carga viral podem resultar em infecções oportunistas, causadas quando o sistema de defesa do organismo está debilitado, como no caso do HIV. A infecção pelo HIV pode ser dividida em quatro fases clínicas: infecção aguda; fase assintomática, também conhecida como latência clínica; fase sintomática inicial ou precoce e a aids ${ }^{14}$. Cada uma dessas fases implica condutas clínicas específicas com igual importância para as perspectivas futuras das PVHA, mas a sua discriminação tem no acompanhamento imunológico um indicador destacado. 
O estímulo para a realização de atividades físicas e a orientação nutricional são também elementos constitutivos do tratamento clínico e físico da PVHA, além de recomendações para a adoção de um estilo de vida saudável, evitando o consumo de álcool e outras drogas que influenciam no CD4 e na carga viral, além dos riscos de interação com os antirretrovirais $(\mathrm{ARV})^{14}$.

\section{O tratamento medicamentoso}

Otratamento das PVHA é focado pelos profissionais de saúde, quase que exclusivamente, nos medicamentos, o que coloca a adesão como ponto principal do desenlace da patologia, bem como das suas dificuldades.

Desta forma, a adesão ao tratamento medicamentoso e às estratégias de ocultação do diagnóstico são desafios enfrentados pelos profissionais da saúde, colocando a terapêutica medicamentosa em destaque na construção psicossocial da doença e do próprio tratamento.

A grande problemática nossa é a adesão medicamentosa. (E4)

Esses doentes que, às vezes, têm que tomar uns 20 comprimidos por dia, quando para de sentir as coisas, para de fazer tudo. Tem que estar sentindo para tomar remédio, quando deixa de sentir para o remédio. Eles deixam de tomar, para quem está em casa não saber, arrancam o rótulo para família não saber. (E6)

A análise dos determinantes da adesão ao tratamento é complexa, uma vez que envolve uma trama de fatores que contribuem para a sua descontinuidade ou para o seu abandono. Alguns fatores envolvidos na não adesão foram identificados pelos participantes desta pesquisa, fatores estes relacionados à apresentação dos medicamentos, à pessoa em tratamento, à doença, ao serviço de saúde e ao suporte econômico-social.

Estes achados foram encontrados também em outros estudos ${ }^{15-19}$ e denotam a importância da atuação em equipe junto às PVHA. Estudoconstatou a existência de períodos mais favoráveis para o abandono do tratamento, como a fase inicial, identificando fatores associados como falta de uma rede social de apoio, dificuldades socioeconômicas e as relacionadas ao uso dos antirretrovirais, características relacionadas aos hábitos de vida, como uso de drogas e antecedentes psiquiátricos ${ }^{15}$.

A ausência de sinais ou sintomas foi referida como associada ao abandono do tratamento, assim como a existência de sujeitos que não utilizam a medicação e não revelam esse fato à equipe de saúde, revelando a variedade de comportamentos desencadeados pela doença e pelo contexto psicossocial.

A existência de dificuldades e motivações para a adesão à TARV foi analisada observando-se que a conciliação entre as exigências do tratamento e atividades cotidianas, representações do HIV e TARV, e aquelas inerentes à complexidade do tratamento são fatores que dificultam a adesão. A lembrança dos sintomas da aids, a percepção do aumento da sobrevida e o vínculo com membros da rede social da PVHA são descritos como facilitadores para adesão ao tratamento ${ }^{16}$.

\section{Tratamento psicoemocional-espiritual}

O medo da morte é relatado como um sentimento persistente nas PVHA, o que produz estresse e manifestações psíquicas, além da busca de apoio em entidades divinas. A auto e heterodiscriminação são responsáveis pelo sofrimento desses sujeitos e das suas famílias, implicando necessidade de ações profissionais que apoiem seu enfrentamento.

É atentar para o lado psicológico. Se a pessoa está psicologicamente abalada, vamos pelo menos manter o equilíbrio. (E18)

Tento, de uma forma ou de outra, promover o atendimento espiritual, já vivenciei isso com uma pessoa muito querida, eu sei da importância dessa parte espiritual para o paciente. (E17)

Diminuir o impacto do estigma e da discriminação relacionados ao HIV/AIDS e melhorar a assistência é uma das metas do setor saúde. Para diminuir o sofrimento e o abalo emocional, foram citadas pelos sujeitos como estratégias de superação e enfrentamento a melhoria da autoestima, o respeito à individualidade $\mathrm{e}$ privacidade, o estímulo do desenvolvimento de práticas religiosas e ações de reforço da espiritualidade.

Existem evidências de que a religiosidade e a espiritualidade estão associadas com o bem-estar psicológico e são consideradas fundamentais para as pessoas em momentos de sofrimento e doença grave, traduzindo-se em força para suportar as dificuldades ${ }^{20}$.

A assistência com abordagem biopsicossocial e espiritual do ser humano pode permitir ampliar as práticas de cuidado de uma dimensão estritamente física para outra mais integral. Essa prática de cuidado deve ser direcionada para a mente-corpo-espírito, e não apenas para o vírus, a doença ou o corpo doente, refletindo a reciprocidade das relações humanas, essenciais a uma perspectiva de cuidado integral direcionado à PVHA.

\section{O cuidado relacional}

A dimensão relacional do cuidado, destacada pelos enfermeiros, é expressada na dinâmica das relações estabelecidas entre a PVHA e os profissionais de saúde, em que os atores envolvidos são capazes de influenciar as múltiplas interações presentes na dinâmica do cuidado. Sabe-se que o sucesso do enfrentamento do HIV/AIDS depende de diversos fatores, dentre eles o acolhimento e escuta ativa do cliente pela equipe multidisciplinar, o vínculo com os profissionais de saúde, equipe e serviço de saúde, além do apoio social mais amplo 7,14,21,22. 
A interação, o acolhimento, a atenção e o apoio são ações fundamentais que resultam em uma relação de confiança entre profissional e o cliente, conforme apontam os enfermeiros.

E não basta só a medicação, não basta só dar o banho, mas também tem que interagir com ele naquele momento. (E13).

Eles têm uma grande confiança na gente... Já conhece o paciente, sabe de todas as implicações, até por causa dessa relação de confiança. (E3)

Quando o cuidado contempla a atenção e a presença física, o cuidador não mais realiza procedimentos em alguém, mas uma ação integrada, com envolvimento, dedicação e responsabilidade, proporcionando crescimento para os envolvidos e respeito mútuo na relação do cuidado ${ }^{23-25}$. $\mathrm{O}$ acolhimento permite a introdução de uma relação empática no atendimento, possibilitando a criação de um espaço dialógico e de participação no tratamento ${ }^{26}$.

A complexidade do tratamento das PVHA requer um cuidado essencialmente relacional, considerando seus elementos técnicos e psicossociais, pautado na simetria da relação entre pessoas, valorizando e reconhecendo as singularidades do cliente e do profissional ${ }^{12,27}$.

As tecnologias na área da saúde devem incorporar a noção de tecnologias leves que se expressam como processo de produção da comunicação, das relações, de vínculos que conduzem ao encontro do usuário com as necessidades de ações de saúde ${ }^{28}$.

\section{Terapêutica social}

A AIDS é um fenômeno reconhecidamente de natureza social, acompanhado de processos de segregação baseados em estigmas socialmente construídos e intimamente ligados às representações sociais da doença $^{29}$. Esse aspecto da doença traz sofrimento e compromete a qualidade de vida das PVHA, impondo limitações às relações afetivas, profissionais e aos relacionamentos cotidianos ${ }^{30}$.

As dificuldades econômicas, as especificidades dos diferentes grupos acometidos pela doença e pelo vírus e a necessidade de receberem apoio social são reconhecidos como modalidades de cuidado pelos entrevistados.

O financeiro também, porque como ele vai se prevenir e manter as estruturas do organismo normais se não tem nem o que comer. Dorme mal, come mal, como vai se prevenir? Agora com um poder aquisitivo melhor... (E13)

Pessoas que têm mais anos de estudo, talvez sejam mais difíceis ao serem abordadas pelos profissionais. Outra que vêm de uma classe social menos favorecida, talvez sejam mais vítimas de preconceito. (E1) trabalho e as condições econômicas são questões essenciais na vida das PVHA, podendo interferir no tratamento e na qualidade de vida. As PVHA frequentemente encontram dificuldades em manter a produtividade no trabalho, por necessitar ausentar-se do emprego para as frequentes visitas ao serviço de saúde, pelos efeitos colaterais da TARV e pelo agravamento do seu quadro ${ }^{31}$. As dificuldades para manter o emprego e se reconhecer como sujeitos produtivos socialmente são reconhecidas como integrantes das limitações sociais associadas à doença, acarretando a necessidade de apoio especializado para o seu enfrentamento.

Outro fator que influencia no tratamento das PVHA é o nível de escolaridade, reconhecendo-se que indivíduos com baixo nível de escolaridade tendem a sofrer maior preconceito e apresentar maiores dificuldades para receber apoio emocional ${ }^{32}$. $\mathrm{O}$ apoio e suporte social são fundamentais para a qualidade de vida das PVHA, por contribuir para a adaptação por meio do manejo das emoções, de orientação afetiva e cognitiva e retroinformação, reduzindo o isolamento e aumentando a satisfação com a vida ${ }^{33}$.

\section{Ações terapêuticas educativas}

A educação em/para a saúde é reconhecida como indispensável na prevenção da infecção, para controlar a doença, recuperar a saúde e restabelecer o autocuidado no contexto do HIV/AIDS. Esse é um cuidado voltado ao esclarecimento e orientação desenvolvido na cotidianidade institucional, e permite um espaço dialógico e de interação entre clientes e profissionais de saúde.

Compreende-se o educar como uma forma de cuidar, que deve ser desenvolvido por meio da educação dialógica objetivando habilitar o cliente para o próprio cuidado, além do fortalecimento da autonomia $^{34}$. Constatou-se, neste estudo, a necessidade do cuidado educativo como parte do conjunto de ações essenciais para o enfrentamento da doença.

Na nossa prática, por ser profissional de saúde, a gente se preocupa muito e tenta, sempre que tem oportunidade, orientar sobre a prevenção, pensando até no cenário geral da doença como um todo, e na expansão da doença no mundo. (E12)

Tentar passar mais alguns conhecimentos, saber dele o que ele sabe da doença, como se cuida, como se alimenta, pra você tentar ver se ele melhora e não pegue mais infecções oportunistas. (E13)

Nos discursos, nota-se que o cuidado não deve ser automático ou protocolar sendo necessário conhecer as vivências individuais e coletivas dos sujeitos e seu momento na vida ${ }^{35}$. A prevenção da infecção pelo vírus ou os cuidados com a doença instalada são reconhecidos como objetos das práticas educativas. 
A prática da educação em/para a saúde constitui- se numa das facetas do cuidado no âmbito do HIV/ AIDS, sendo fundamental para a prevenção e para a qualidade de vida dos indivíduos, portadores ou não do vírus. É preciso, no entanto, que esse cuidado seja adequado à realidade sociocultural dos clientes, e que permita a participação e a autorresponsabilização dos indivíduos nesse processo.

\section{Conclusão}

Diante das evoluções técnico-científicas e farmacológicas da TARV, a sobrevida das PVHA tem aumentado, fazendo com que uma síndrome, representada como morte, passasse a ter características de cronicidade. Ainda incurável, a AIDS apresenta possibilidades efetivas de controle, trazendo novos desafios para os profissionais de saúde.

Os conteúdos da representação social do tratamento das PVHA vêm sofrendo transformações, acompanhando a própria evolução da doença e do vírus incorporando, além dos cuidados clínicos e físicos e do tratamento medicamentoso, o cuidado relacional, as ações educativas, a perspectiva psicoemocional- espiritual e a terapêutica social. Essa representação ultrapassou o caráter biológico do tratamento e atingiu aqueles mais afeitos à pessoa humana, considerando que o HIV/AIDS ocasiona inúmeras consequências psicoespirituais, sociais, profissionais e familiares, abarcando o ser humano em sua integralidade e repercutindo em vários aspectos da vida.

Constatou-se, a partir deste estudo, uma amplitude de ações implicadas em diferentes modalidades de tratamento e, portanto, de cuidado das PVHA, não restritas ao tratamento medicamentoso e clínico, mas envolvendo um conjunto de necessidades físicas, psicológicas, espirituais, sociais, educativas, familiares e relacionais. Observou-se uma rede de representações associadas ao tratamento das PVHA que, ao mesmo tempo em que aponta ações reconhecidas como parte do controle da doença, se volta para o alcance da qualidade de vida e para o futuro das PVHA e da própria sociedade.

O presente estudo apresenta como limitações o quantitativo de profissionais investigados e o intervalo de tempo decorrido desde sua realização, todavia considerando o cenário mundial e nacional da AIDS, acredita-se que esses achados sejam relevantes para a pesquisa na área de enfermagem e do cuidado a PVHA.

\section{REFERÊNCIAS}

1.Ministério da Saúde (Br). Secretaria de Vigilância em Saúde. Departamento de Aids e Hepatites Virais. Boletim Epidemiológico DST/Aids 2014. Brasília (DF): Ministério da Saúde; 2014. [citado em 10 jul 2015]. Disponível em: http://www.aids.gov.br/sites/default/files/anexos/publica- cao/2014/56677/boletim2014finalpdf156655.pdf 2.Navarro AMA, Bezerra VP, Oliveira DA, Moreira MASP, Alves MSCF, Gurgel SN. Social Representations of the HIV/AIDS: perception of the primary health care professionals. R pesq.: cuid fundam. [online] 2011; (Supl):92-9. 3.Hall R, Ravasi G, Kuchenbecker R, Greco D, Simão M. $\mathrm{O}$ acesso universal ao tratamento antirretroviral no Brasil. Tempus actas de saúde colet. 2010; 4:53-65.

4.Souza S. Os discursos de adesão ao tratamento de AIDS - uma reflexão ética. Rev Bioethikos - Centro Universitário São Camilo. 2011; 5:98-106.

5.Luz PM, Miranda KCL. As bases filosóficas e históricas do cuidado e a convocação de parceiros sexuais em HIV/ aids como forma de cuidar. Ciênc saúde coletiva. 2010; 15:1143-8.

6.Formozo GA, Oliveira DC. Representações sociais do cuidado prestado aos pacientes soropositivos ao HIV. Rev Bras Enferm. 2010; 63:230-7.

7.Souza CSO, Silva AL. O cuidado a pessoas com HIV/ aids na perspectiva de profissionais de saúde. Rev esc enferm USP. 2013; 47:907-14.

8. Oliveira DC, Gomes AMT, Pontes APM, Costa CPM. Construção de um paradigma de cuidado de enfermagem pautado nas necessidades humanas e de saúde. Esc Anna Nery. 2011; 15:838-44.

9.Gonçalves RBM. Práticas de saúde: processos de trabalho e necessidades. São Paulo: CEFOR; 1992.

10.Moscovici S. Representações sociais: investigações em psicologia social. Petrópolis(RJ): Vozes; 2003.

11.Jodelet D. organizadora. As Representações Sociais. Rio de Janeiro: EdUERJ; 2001.

12.Bardin L. Análise de Conteúdo. Lisboa(Pt): Ed. 70; 2007.

13. Oliveira DC. Análise de conteúdo temática: uma proposta de sistematização. Rev enferm UERJ. 2008; 16:569-76.

14.Ministério da Saúde (Br). Secretaria de Vigilância em Saúde. Departamento de Aids e Hepatites Virais. Protocolo clínico e diretrizes terapêuticas para manejo da infecção pelo HIV em adultos. Brasília (DF): Ministério da Saúde; 2013. [citado em 10 jul 2015]. Disponível em: http://bvsms.saude.gov.br/bvs/publicacoes/protocolo_clinico manejo hiv adultos.pdf.

15.Schilkowsky L $\bar{B}$, Portela MC, Sá MC. Fatores associados ao abandono de acompanhamento ambulatorial em um serviço de assistência especializada em HIV/aids na cidade do Rio de Janeiro, RJ. Rev bras epidemiol. 2011; 14:187-97.

16.Santos WJ, Drumond EF, Gomes AS, Corrêa CM, Freitas MIF. Barreiras e aspectos facilitadores da adesão à terapia antirretroviral em Belo-Horizonte - MG. Rev Bras Enferm. 2011; 64:1028-37.

17.Beyene KA, Gedif T, Gebre-Marian T, Engidawork E. Highly active antiretroviral therapy adherence and its determinants in selected hospitals from south and central Ethiopia. Pharmacoepidemiology And Drug Safety. 2009; 18:1007-15.

18.Roura M, Busza J, Wringe A, Mbata D, Urassa M, Zaba B. Barriers to Sustaining Antiretroviral Treatment in Kisesa, Tanzania: A Follow-Up Study to Understand 
Attrition from the Antiretroviral Program AIDS. Patient care and STDs. 2009; 23:203-10.

19.Bonolo PF, Machado CJ, Cesar CC, Ceccato MGB, Guimaraes MDC. Vulnerability and non-adherence to antiretroviral therapy among HIV patients, Minas Gerais State, Brazil. Cad Saúde Pública. 2008; 24:2603-13.

20.Fleck MPA, Borges ZN, Bolognesi G, Rocha NS. Desenvolvimento do WHOQOL módulo espiritualidade, religiosidade e crenças pessoais. Rev Saude Publica. 2003; 37:446-55.

21.Neves CLR, Amorim WM, Moraes NA, Leite JL. The nursing care of the client with hiv/aids in a university hospital in the 1980's. R pesq: cuid fundam. 2009; 1:299-316 22.Monteiro JFA, Figueiredo MAC. Vivência profissional: subsídios à atuação em HIV/AIDS. Paidéia. 2009; 19:67-76.

23.Ferreira M A. O corpo no cuidado de enfermagem: representações de clientes hospitalizados [tese de doutorado]. Rio de Janeiro: Universidade Federal do Rio de Janeiro; 1999. 24. Waldow VR. Cuidado humano: o resgate necessário. $2^{\text {a }}$ ed. Porto Alegre (RS): Sagra D.C. Luzzatto; 1999.

25. Barcelos LMS, Alvim NAT. Atenção e presença física: dimensões expressivas e a prática dialógica do cuidado de enfermagem na perspectiva do cliente hospitalizado. Rev Bras Enferm. 2006; 59:25-9.

26.Silva NEK, Oliveira LA, Figueiredo WS, Landroni MAS, Waldman CCS, Ayres JRCM. Limites do trabalho multiprofissional: estudo de caso dos centros de referência para DST/AIDS. Rev Saude Publica. 2002; 36:108-16.
27.Santos EI, Gomes AMT, Oliveira DC, Santo CCE. Entre sofrimento e prazer - a vulnerabilidade para enfermeiros nas relações interpessoais com paciente com HIV/ AIDS. Rev enferm UERJ. 2013; 21:9-15.

28.Merhy EE, Franco TB. Por uma composição técnica do trabalho em saúde centrada no campo relacional e nas tecnologias leves: apontando mudanças para os modelos tecno-assistenciais. Saúde debate. 2003; 27:316-23.

29. Malbergier A. AIDS e psiquiatria: um guia para os profissionais de saúde. Rio de Janeiro: Revinter; 2000.

30.Sousa MMO. SIDA: e a vida continua. Lisboa (Pt): Associação Portuguesa de Enfermeiros; 2001.

31.Tunala LG. Fontes cotidianas de estresse entre mulheres portadoras de HIV Rev Saude Publica. 2002; 36:24-31.

32.Seidl EMF, Zannon CMLC, Troccoli BT. Pessoas Vivendo com HIV/AIDS: Enfrentamento, suporte social e qualidade de vida. Psicol Reflex Crit. 2005; 18:188-95. 33.Resende MC, Bones VM, Souza IS, Guimaraes NK. Rede de relações sociais e satisfação com a vida de adultos e idosos. Psicol Am Lat. [Internet]. 2006; 5:0-0 [citado em 24 set 2014]. Disponível em: http://pepsic. bvsalud.org/scielo.php?script $=$ sci_arttext\&pid $=$ S1870. $-350 \times 2006000100015 \& \operatorname{lng}=\mathrm{pt} \& \bar{n} r \mathrm{~m}=$ iso.

34.Alves IC, Padilha MICS, Mancia JR. A equipe de enfermagem e o exercício do cuidado a clientes portadores de HIV/AIDS. Rev enferm UERJ. 2004; 12:133-9.

35.Paiva V, Latorre MR, Gravato N, Lacerda R. Sexualidade de mulheres vivendo com HIV/AIDS em São Paulo. Cad Saúde Pública. 2002; 18:1609-20. 